\title{
The staining pattern of human sperm with Diff Quik: relationship with sperm head morphology and a sperm chromatin structure assay (SCSA)
}

Natalie Moska ${ }^{1,2}$, Elaine Murray' ${ }^{2}$ Penelope Wakefield ${ }^{2}$, Phillip Matson ${ }^{3}$

${ }^{2}$ Hollywood Fertility Centre, Nedlands, Western Australia, ${ }^{3}$ Murdoch University, Murdoch, Western Australia, Australia

Received: 5 June 2010; accepted: 19 November 2010

\section{SUMMARY}

The dark staining of human sperm heads by Diff Quik is significantly correlated with abnormal sperm head morphology $(\mathrm{r}=0.51, \mathrm{p}<0.0001)$, but is not associated with changes in sperm chromatin detected by a sperm chromatin structure assay (SCSA; $r=0.18, p>0.09$ ). Whilst valuable in the assessment of head morphology, it is concluded that Diff Quik staining is not a useful substitute for the SCSA to assess human sperm chromatin. Reproductive Biology 201111 1: 55-59.

Key words: Diff Quik, sperm chromatin, DNA fragmentation index

${ }^{1}$ Corresponding author: Hollywood Fertility Centre, Hollywood Private Hospital, Nedlands 6009, Western Australia 6009, Australia; e-mail: natalie.moska@hollywoodivf.com

Copyright (C) 2011 by the Society for Biology of Reproduction 


\section{INTRODUCTION}

There are a number of elaborate tests that can be used to examine sperm chromatin and the contained DNA, using technology that assesses DNA damage either indirectly [e.g. the sperm chromatin structure assay (SCSA) after mild acidic denaturation] or directly (e.g. the comet and TUNEL assays; [12]). Whilst methods for evaluating sperm DNA integrity are thought by some to have poor clinical utility [9], the need to identify sperm with damaged DNA is still regarded as a priority [7]. The SCSA appears to be a well described technique [4] which has shown good predictive value for the outcome of assisted reproduction [1]. A simpler and cheaper method of staining sperm with Diff Quik has been proposed for the evaluation of sperm chromatin for both humans [8] and felids [6]. This is based upon the intensity of nuclear staining, with sperm heads containing fragmented or damaged DNA showing a dark blue staining pattern due to an alteration in the thiazine-DNA interaction [8].

The assessment of both sperm morphology and chromatin on the same slide would be attractive to andrology laboratories. The aim of the current study was therefore to investigate further the use of Diff Quik in assessing human sperm heads by (i) determining the relationship between sperm head morphology and the Diff Quik staining pattern, and (ii) comparing the results of the stain with an SCSA used to assess sperm chromatin.

\section{MATERIALS AND METHODS}

Men attending the Hollywood Fertility Centre produced a semen sample as part of their routine investigation of infertility. Semen samples were collected by masturbation after a period of sexual abstinence of 2-5 days, and processed within one hr for volume, and sperm concentration, morphology and motility [11]. Slides were made by applying a $10 \mu \mathrm{l}$ drop of semen to a microscope slide and a smear air-dried. They were then stained with Diff Quik (Lab Aids Pty Ltd, North Narrabeen, NSW 2101, Australia; [5]) and stored. Slides were removed from storage and sperm observed through 
immersion oil with bright field illumination at 1000 times magnification and the criteria of sperm head normality was that described in the WHO manual third edition [10]. The staining pattern of the head was also noted as being either pale or dark blue [8]. Individual sperm were therefore categorized as (a) normal head morphology and light staining, (b) normal head morphology and dark staining, (c) abnormal head morphology and light staining, and (d) abnormal head morphology and dark staining. An SCSA was performed as described elsewhere [3] on semen snap frozen in liquid nitrogen at the time of analysis. A DNA fragmentation index (DFI) was calculated as the proportion of sperm with an elevated ratio of single to double stranded DNA compared to the main cell population.

Data were analyzed using StatistiXL (Nedlands, Western Australia 6009, Australia). Group data were expressed as median (range) with comparisons made using the Mann-Whitney $U$ test, and correlations were tested with the null hypothesis that the observed value was from a population in which the true correlation was zero. All differences were considered significant if $\mathrm{p}<0.05$.

\section{RESULTS AND DISCUSSION}

The relationship between staining and sperm head morphology for individual sperm was assessed by analyzing 71 stained semen smears, and the proportion of heads stained dark was significantly higher $(U=5021.0 ; p=0.000)$ for morphologically abnormal sperm $[23.9 \%(6.9 \%-54.3 \%)]$ than normal sperm [0.0\% (0.0\%-16.7\%)]. Accordingly, a significant correlation between abnormal morphology and dark staining existed $(\mathrm{r}=0.51, \mathrm{p}<0.0001)$ as shown in Figure 1. However, a poor correlation existed between the proportion of sperm staining dark and the DFI obtained using an SCSA $(r=0.18, n=53$, $\mathrm{p}>0.09$ ) which is in contrast to a significant correlation of 0.68 between the staining and fragmentation of DNA detected by the TUNEL assay [8]. Given there is a generally a good agreement between the TUNEL and SCSA methods [2], our failure to see the same relationship between the Diff Quik staining and the SCSA result was disappointing. Given that the SCSA 


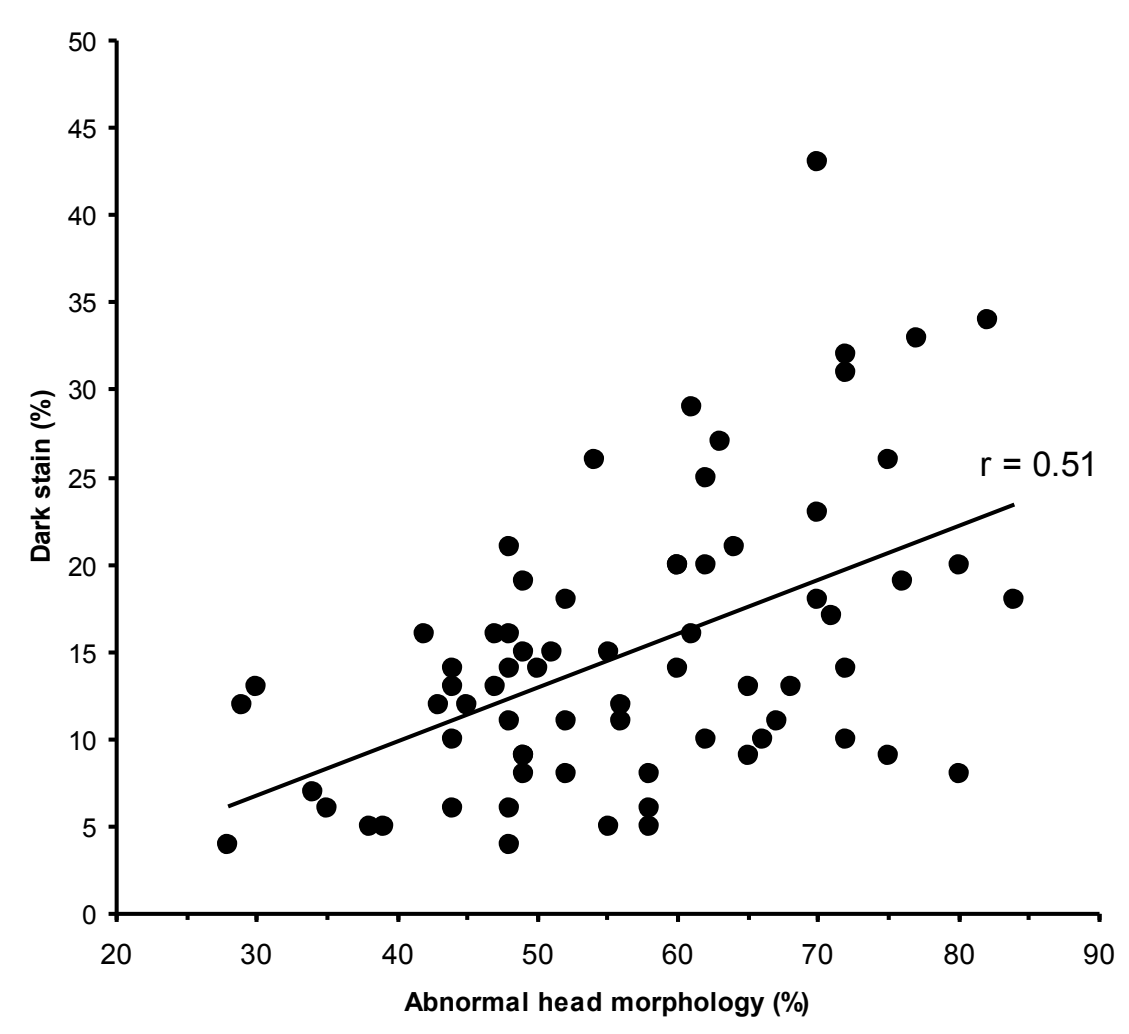

Figure 1. The correlation between the proportion of sperm with abnormal head morphology and the proportion of sperm heads staining dark with Diff Quik for 71 semen samples from different men.

and TUNEL methods are very different in their underlying principal [7], it is tempting to suggest that the TUNEL and the stain are therefore both detecting similar damage directly which was not detected by the indirect SCSA. However, the original authors did note in their report when discussing the TUNEL and stain techniques that "the two methods ... do not measure the same parameter" [8]. The point was also made by the authors that the time required for the Diff Quik staining appears critical and is variable between brands and batches of Diff Quik [8]. It may be that our conditions were not optimal to get the same results, but it still remains that a method so subjective in its operation would be of limited benefit in a laboratory with 
an established Diff Quik staining protocol for morphology assessment but looking for a simple and robust alternative to the SCSA method.

It is concluded that the dark staining seen with Diff Quik is largely limited to sperm with abnormal head morphology, and is not associated with changes in sperm chromatin detected by the SCSA. Diff Quik staining is therefore not a useful alternative to the SCSA.

\section{REFERENCES}

1. Bungum M, Humaidan P, Spano M, Jepson K, Bungum L, Giwercman A 2004 The predictive value of sperm chromatin structure assay (SCSA) parameters for the outcome of intrauterine insemination, IVF and ICSI. Human Reproduction 19 1401-1408.

2. Chohan K, Griffin J, Lafromboise M, De Jong C, Carrell D 2006 Comparison of chromatin assays for DNA fragmentation evaluation in human sperm. Journal of Andrology 27 53-59.

3. Evenson D, Jost L $\mathbf{2 0 0 0}$ Sperm chromatin structure assay is useful for fertility assessment. Methods in Cell Science 22 169-189.

4. Evenson D, Larson K, Jost L $\mathbf{2 0 0 2}$ Sperm chromatin structure assay: its clinical use for detecting sperm DNA fragmentation in male infertility and comparisons with other techniques. Journal of Andrology 23 25-43.

5. Kruger TF, Ackerman SB, Simmons KE, Swanson RJ, Brugo SS, Acosta AA 1987 A quick, reliable staining technique for human sperm morphology. Archives of Andrology $18275-277$.

6. Mota P, Ramalho-Santos J 2006 Comparison between different markers for sperm quality in the cat: Diff-Quik as a simple optical technique to assess changes in the DNA of feline epididymal sperm. Theriogenology 65 1360-1375.

7. Sakkas D, Alvarez J 2010 Sperm DNA fragmentation: mechanisms of origin, impact on reproductive outcome, and analysis. Fertility and Sterility 93 1027-1036.

8. Sousa A, Tavares R, Velez de la Calle J, Figueiredo H, Almeida V, Almeida-Santos T, Ramalho-Santos J 2009 Dual use of Diff-Quik-like stains for the simultaneous evaluation of human sperm morphology and chromatin status. Human Reproduction $\mathbf{2 4}$ $28-36$.

9. The Practice Committee of the American Society for Reproductive Medicine 2008 The clinical utility of sperm DNA integrity testing. Fertility and Sterility 90 S178-S180.

10. WHO 1992 WHO laboratory manual for the examination of human semen and semencervical mucus interaction., 3rd Edition. Cambridge University press, Cambridge.

11. WHO 2010 WHO laboratory manual for the examination and processing of human semen., 5th Edition. Cambridge University Press, Cambridge.

12. Zini A, Sigman M 2009 Are tests of sperm DNA damage clinically useful? Pros and cons. Journal of Andrology 30 219-229. 